Article

\title{
Effect of Surface Topography on Particle Deposition from Liquid Suspensions in Channel Flow
}

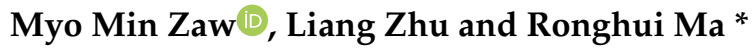 \\ Department of Mechanical Engineering, University of Maryland, Baltimore County, 1000 Hilltop Circle, \\ Baltimore, MD 21250, USA; myo1@umbc.edu (M.M.Z.); zliang@umbc.edu (L.Z.) \\ * Correspondence: roma@umbc.edu
}

Received: 9 November 2019; Accepted: 3 January 2020; Published: 5 January 2020

\begin{abstract}
A Eulerian-Lagrangian model has been developed to simulate particle attachment to surfaces with arc-shaped ribs in a two-dimensional channel flow at low Reynolds numbers. Numerical simulation has been performed to improve the quantitative understanding of how rib geometries enhance shear rates and particle-surface interact for various particle sizes and flow velocities. The enhanced shear rate is attributed to the wavy flows that develop over the ribbed surface and the weak vortices that form between adjacent ribs. Varying pitch-to-height ratio can alter the amplitude of the wavy flow and the angle of attack of the fluid on the ribs. In the presence of these two competing factors, the rib geometry with a pitch-to-height ratio of two demonstrates the greatest shear rate and the lowest fraction of particle attachment. However, the ribbed surfaces have negligible effects on small particles at low velocities. A force analysis identifies a threshold shear rate to reduce particle attachment. The simulated particle distributions over the ribbed surfaces are highly non-uniform for larger particles at higher velocities. The understanding of the effect of surface topography on particle attachment will benefit the design of surface textures for mitigating particulate fouling in a wide range of applications.
\end{abstract}

Keywords: surface topography; particle attachment; particle surface interactions; Eulerian-Lagrangian simulation

\section{Introduction}

Understanding deposition of particles from liquid or gas suspensions on a solid surface is important in numerous engineering applications, such as oil refineries, food and pharmaceutical industries, and environmental science. Three mechanisms have been identified in the deposition process: particle transport from bulk flow to the surface, particle attachment on the surface, and particle re-entrainment from the surface [1-4]. Depending on particle sizes, particle transport can be divided into three regimes: diffusion, inertia, and impact [2,4]. Near the surface, particle attachment and resuspension are determined by the interplay among adhesive particle-surface interactions, hydrodynamic drag, lift force, gravity, surface roughness, etc. Among many factors, surface topography, such as random roughness, grooves, and ribs, can change contact geometries and hydrodynamics near the surface, thus, having substantial influence on the particle deposition [5]. While extensive research effort has been made towards understanding the effect of random or designed surface roughness on particle deposition from gas flows [4], particle interactions with surface topography in liquid suspensions are less addressed, despite the considerably great hydrodynamic lift force near the wall [2].

A case of interest is ribbed surfaces for heat exchangers. Developed for enhancing heat transfer [6-9] and/or mitigating crystallization fouling $[10,11]$, these structures may exacerbate particulate fouling. Assuming an analogy between particle transport and heat transfer, theoretical studies $[6,12]$ suggest that rib structures intensify particle transport to the surface, but, in the meantime, the enhanced local 
shear rate reduces the fraction of the particles sticking to the surface. Thereby, it is possible to achieve both enhanced heat transfer and mitigated particle deposition with surface structures [12]. However, this conclusion is not completely supported by the experimental studies. Kim and Webb [6] studied particulate fouling of three types of ribbed tubes and a smooth tube for 14,000 $<R e<26,000$ using ferric oxide particles ( 0.64 micron in diameter) and aluminum oxide particles ( 3 microns in diameter) dispersed in water. All ribbed tubes were found to increase particulate fouling with the only exception of fouling at a high $\operatorname{Re}(26,000)$ where the extent of fouling was approximately the same as the smooth tube. Based on the experimental study, the first accelerated particulate fouling model was developed for helically ribbed tubes. Somerscales et al. [8,9] reviewed the particulate fouling characteristics of five different in-tube heat transfer enhancement surfaces. Their study suggests that certain surface geometries (e.g., roped surfaces) are effective in battling particulate fouling especially at high velocities. Li [13] studied particulate fouling of helically ribbed surfaces using an accelerated particle fouling test. More particle deposition occurs on all ribbed surfaces with different geometrical parameters at a Reynolds number around 16,000. More review on experimental studies of particulate fouling of surfaces with structures for heat transfer enhancement can be found in the References [7-9]. Textured surfaces used to suppress attachment of bio-organisms through enhanced shear [14] are out of the scope of this work, and thus, are not reviewed.

The differential behaviors of particle deposition near surface structures in previous studies [6-9] reveal the complicated interplay among particles, fluid flow, and surface topography. As an alternative to experimental studies, computational models of various complexities have been developed to acquire quantitative understanding of particle attachment to complex surfaces. Lu and Lu [15-18] performed Computational Fluid Dynamics (CFD) simulations of particle deposition from an air flow on three different surfaces with circular, triangular, and square ribs, respectively. Their studies suggest that particle deposition increases with short rib spacing and increased rib height, although the latter has less influence. Most particles accumulate on the windward area of the ribs. Among the three different rib shapes, the circular shape demonstrates the least particle deposition, while the square-shaped rib has the highest. Hong et al. [19] conducted a two-dimensional numerical study on particle deposition on structured elements from gas flow. The range of the height and the spacing of the elements are $0.4 \sim 2 \mathrm{~mm}$ and $4 \sim 16 \mathrm{~mm}$, respectively, and the diameter of the channel is $20 \mathrm{~mm}$. This study shows more particle deposition on surfaces with increased element height and spacing. $\mathrm{Xu}$ et al. [20] studied the submicron particle deposition on a semi-circular structured surface and suggested that the recirculation wake is the main mechanism for more particle deposition. Kasper et al. [21,22] developed numerical models with the capability of studying particle deposition on structured surfaces from turbulent liquid flow, and the predicted particle deposition agrees with their experimental study. More computational studies for particle transport and deposition are given in the review paper by Guha [4]. Compared with computational studies of gas suspensions, particle deposition from liquid suspensions on surfaces with various topographies are less-frequently addressed, especially for low-Reynolds-number flows.

In this paper, a Eulerian-Lagrangian model is developed to simulate particle attachment to surfaces with arc-shaped ribs in a two-dimensional channel flow at low Reynolds numbers. Numerical simulations are performed to improve the quantitative understanding of how rib geometries enhance shear rates and particle-surface interactions for various particle sizes and flow velocities. Of special interest is how the geometrical parameters of these ribs, such as pitch-to-height ratios, modify the hydrodynamics and particle-surface interactions for various particle sizes and flow velocities. A force analysis is performed to identify a threshold shear rate for reduction of particle attachment. The model is also used to predict particle distributions over the ribbed surfaces for different particle sizes and flow velocities. It is anticipated that the understanding of the effect of surface topography on particle attachment will benefit design of surface textures for mitigating particulate fouling in a wide range of applications. 


\section{Mathematical Models}

We develop a Eulerian-Lagrangian model to describe the attachment of micrometer-sized particles on surfaces from liquid suspensions moving at low Reynolds numbers. Assuming a low volumetric particle concentration $(<5 \%)$, the liquid suspension is treated as an incompressible and Newtonian fluid. The effects of particles and particle-particle collisions on the fluid flow are considered negligible. The dynamics of the fluid flow is described by the mass conservation and the Navier-Stokes equations, which are:

$$
\begin{gathered}
\nabla \cdot \mathbf{u}_{\mathbf{f}}=0 \\
\rho_{f} \frac{\partial \mathbf{u}_{\mathbf{f}}}{\partial t}+\nabla \cdot\left(\rho_{f} \mathbf{u}_{\mathbf{f}} \mathbf{u}_{\mathbf{f}}\right)=-\nabla P+\nabla \cdot\left[\mu_{f} \nabla\left(\mathbf{u}_{\mathbf{f}}+\mathbf{u}_{\mathbf{f}}{ }^{\mathbf{T}}\right)\right]+\rho_{f} \mathbf{g}
\end{gathered}
$$

where $\mathbf{u}_{\mathbf{f}}$ is the fluid velocity vector, $\rho_{f}$ is the fluid density, $\mu_{f}$ is the fluid viscosity, $P$ is the pressure, and $\mathbf{g}$ is the acceleration due to gravity. The Lagrangian method is used to describe the motion of the particles with diameters in the range of 10 75 microns. The position and velocity of a particle are determined based on Newton's second law of motion:

$$
\frac{d X_{\mathrm{p}, \mathrm{i}}}{d t}=\mathbf{u}_{\mathrm{p}, \mathrm{i}} \text { and } m_{p, i} \frac{d \mathbf{u}_{\mathbf{p} . \mathbf{i}}}{d t}=\sum_{1}^{N} \mathbf{F}_{\mathrm{i}}
$$

where $X_{\mathrm{p}, \mathrm{i}}$ is the position of a particle, $\mathbf{u}_{\mathrm{p}, \mathrm{i}}$ is the particle velocity vector, $m_{p, i}$ is the mass of the particle, $F_{i}$ is the force acting on the particle, and $N$ is the total number of the forces. The particles are assumed to be spherical and rigid. For liquid suspensions passing a channel at low Reynolds numbers, forces acting on the particles are drag, buoyancy, gravity, pressure gradient, Saffman lift force, and wall-induced lift force. As the main focus of this study is the effect of surface topography on particle attachment to a solid surface, particles are assumed to be neutrally charged and the electrostatic double layer force is not included. Brownian force is not considered because its magnitude is relatively weak compared with other forces for the particle size of interest in this study. The acceleration, velocity, and location of a discrete particle are calculated at each time step by numerical integration of Equation (3).

The general form of the drag force $\mathbf{F}_{\mathbf{D}}$ acting on a particle developed for a wide range of Reynolds numbers is expressed in terms of a drag coefficient $C_{D}$, which is [23]:

$$
\mathbf{F}_{\mathbf{D}}=C_{D} \frac{\pi d_{p}^{2}}{8} \rho_{f}\left(\mathbf{u}_{\mathbf{f}}-\mathbf{u}_{\mathbf{p}}\right)\left|\mathbf{u}_{\mathbf{f}}-\mathbf{u}_{\mathbf{p}}\right|
$$

where $d_{p}$ is the diameter of the particle, $\mathbf{u}_{\mathbf{f}}$ is the fluid velocity at the point where the center of a particle is located, $\mathbf{u}_{\mathbf{p}}$ is the particle velocity, and $\left|\mathbf{u}_{\mathbf{f}}-\mathbf{u}_{\mathbf{p}}\right|$ is the magnitude of the relative slip velocity. The drag coefficient $C_{D}$ depends on the particle Reynolds number, which is defined as:

$$
R e_{p}=\frac{\rho_{f} d_{p}}{\mu_{f}}\left|\mathbf{u}_{\mathbf{f}}-\mathbf{u}_{\mathbf{p}}\right|
$$

The correlation for the drag coefficient proposed by Putnam [24] is used in this study due to its suitability for a wide range of particle Reynolds numbers while imposing the correct limiting drag force within the Newtonian regime:

$$
C_{D}=\left\{\begin{array}{c}
\frac{24}{R e_{p}}\left(1+\frac{1}{6} \operatorname{Re}_{p}^{\frac{2}{3}}\right) \text { if } \operatorname{Re}_{p}<1000 \text { (Laminar regime) } \\
0.424 \text { if } \operatorname{Re}_{p} \geq 1000 \text { (Turbulent regime) }
\end{array}\right\} .
$$

While this correlation is limited to smooth particles, the calculated drag force can be extended to rough particles in this study because the effect of surface roughness is negligible under the conditions of laminar flow and low $R e_{p}$, which is the case of the current study. 
The buoyancy and the gravitational forces on a particle are combined as one total force $\mathbf{F}_{g}$ :

$$
\mathbf{F}_{g}=m_{p} \mathbf{g}\left(1-\frac{\rho_{f}}{\rho_{p}}\right)
$$

where $\rho_{p}$ is the density of the particle. The pressure gradient force resulted from local pressure change is important for solid particles suspended in liquid [23]:

$$
\mathbf{F}_{p}=-\frac{\pi d_{p}^{3}}{6} \nabla p
$$

A non-uniform velocity field gives rise to the Saffman lift force in the direction normal to the fluid flow. Mathematical description of this shear induced lift force was first developed by Saffman $[25,26]$ and advanced by Mei [27]. The Saffman lift force $\mathbf{F}_{L, S}$ is expressed as:

$$
\mathbf{F}_{L, S}=C_{L} \frac{\pi D_{p}^{3}}{6} \rho_{f}\left(\mathbf{u}_{\mathbf{f}}-\mathbf{u}_{\mathbf{p}}\right) \times\left(\nabla \times \mathbf{u}_{\mathbf{f}}\right)
$$

where $C_{L}$ is the Saffman lift force coefficient. Formulations to calculate $C_{L}$ can be found in the reference [27].

In addition to the Saffman lift force, a particle moving close to a wall is subject to a viscous lift force because of the progressively increasing friction between the two approaching surfaces. The expression for the wall-induced lift force that is normal to the surface $[28,29]$ is:

$$
\mathbf{F}_{L}=f_{L} \rho_{f} \gamma^{2} d_{p}^{4}
$$

where $f_{L}$ is lift coefficient, and $\gamma$ is local shear rate. The lift coefficient depends on the local Reynolds number [29]:

$$
f_{L}=3.4368 R e^{-0.714}
$$

The adhesive van der Waals force between a spherical particle and a surface has the form of [30-33]

$$
F_{v d w}=\frac{A_{H} d_{p}}{12 z^{2}}
$$

where $A_{H}$ is the Hamaker constant and $z$ is the surface-to-surface distance between the particle and the wall. The Combining Relation method [34] can be used to evaluate $A_{H}$ in Equation (12) based on the experimental and theoretical values of the related materials [35].

Upon contact, a particle may adhere to or bounce back from the surface.

The collision outcome is dependent on the approaching velocity of a particle and the particle-surface interactions. The principle of the conservation of energy has been widely used to describe the states of a particle before and after the particle-wall collision [36]:

$$
E_{k i n, 1}+E_{e l, 1}=E_{v d W}+E_{k i n, 2}+E_{e l, 2}+E_{\text {Loss }}
$$

where $E_{k i n}$ is the kinetic energy, $E_{e l}$ is the electrostatic energy, $E_{v d W}$ is the van der Waals attraction energy between the particle and the surface, and $E_{\text {Loss }}$ is the dissipation energy. $E_{\text {Loss }}$ is resulted from particle deformation and friction during the collision. Subscripts 1 and 2 represent the states before and after a collision, respectively. It is generally accepted that a particle will adhere to the surface if its kinetic energy after the collision is zero, i.e., $E_{k i n, 2}=0[36,37]$. Based on this criterion, the critical approaching velocity $U_{c r}$ for particle adherence can be derived from Equation (13). When the electrostatic energy 
and the energy loss are not considered [37] for neutrally charged and rigid particles, the critical velocity $U_{c r}$ for particle adherence has the form of:

$$
U_{c r}=\sqrt{\frac{12 E_{v d w}}{\pi d_{p}^{3} \rho_{p}}}
$$

Particles approaching the surface at velocities greater than $U_{c r}$ will result in $E_{k i n, 2}>0$, and are predicted to bounce back. The van der Waals energy is calculated by integrating of the product of the van der Waals pressure and the contact area over the distance to the wall [38]. The van der Waal energy of a particle in Equation (14) at the wall is [38]:

$$
E_{v d w}=\frac{A_{H}^{2} d_{p}}{144 \pi z_{0}^{4} \sigma}
$$

where $z_{0}$ is the distance between the particle and the surface upon contact and $\sigma$ is the yield strength of polystyrene particles. The values of $A_{H}, z_{0}$, and $\sigma$ used in this study are given in Table 1.

Table 1. Physical properties and simulation parameters.

\begin{tabular}{cc}
\hline Variable & Value \\
\hline Height of the channel, $h$ & $20 \mathrm{~mm}$ \\
Height of the rib, $H$ & $1 \mathrm{~mm}$ \\
Density of water, $\rho_{f}[39]$ & $998.2 \mathrm{~kg} / \mathrm{m}^{3}$ \\
Viscosity of water, $\mu_{f}[39]$ & $0.001003 \mathrm{~Pa} \mathrm{~s}$ \\
Density of particle, $\rho_{p}$ & $1040 \mathrm{~kg} / \mathrm{m}^{3}$ \\
Contact distance, $z_{0}[41]$ & $40 \mathrm{MPa}$ \\
Yield strength of polystyrene particles, $\sigma[40]$ & $0.4 \mathrm{~nm}$ \\
Hamaker constant, $A_{H}[34,35]$ & $1.69 \times 10^{-20} \mathrm{~J}$ \\
Maximum channel velocity, $u_{\text {max }}$ & $3 \mathrm{~cm} / \mathrm{s}, 8 \mathrm{~cm} / \mathrm{s}$, and $13 \mathrm{~cm} / \mathrm{s}$ \\
Diameter of particles, $d_{p}$ & $10 \mu \mathrm{m}, 50 \mu \mathrm{m}$, and $75 \mu \mathrm{m}$ \\
Critical velocity of particle adherence, $U_{c r}$ & $0.36,0.079,0.048 \mathrm{~cm} / \mathrm{s}$ \\
Channel Reynolds number & $500 \sim 3000$ \\
Particle relaxation time & $5.76 \times 10^{-6} \sim 3.24 \times 10^{-4} \mathrm{~s}$ \\
Particle Reynolds number, $R e_{p}$ & $0.299 \sim 9.70$ \\
\hline
\end{tabular}

\section{Problem Setup and Numerical Issues}

The deposition of polystyrene particles from an aqueous suspension is studied in a two-dimensional copper channel. The channel is $20 \mathrm{~mm}$ in height and $140 \mathrm{~mm}$ in length. As shown in Figure 1a, it has a $20 \mathrm{~mm}$ long entry region, a $100 \mathrm{~mm}$ long test surface with repeated arc-shaped ribs, and a $20 \mathrm{~mm}$ long region for exit. The arc-shaped two-dimensional ribs with identical height but four different pitch-to-height ratios $(\lambda / \mathrm{H})$ are shown in Figure $1 b$.

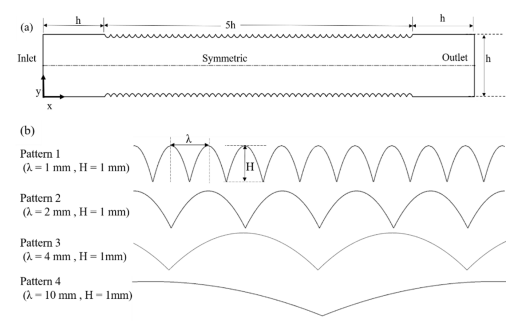

Figure 1. The schematics of (a) the two-dimensional channel and (b) the arc-shaped ribs. 
A fully developed flow with a parabolic velocity profile is applied at the inlet:

$$
u_{\text {inlet }}(y)=\frac{4 u_{\max } y(h-y)}{h^{2}}
$$

where $h$ is the height of the channel and $u_{\max }$ is the maximum inlet velocity. At the outlet, $p=0$. On the channel wall, a no slip condition is applied, which is $\mathbf{u}_{\mathbf{f}}=0$. Due to the symmetry, only half of the domain is simulated, and the symmetric boundary condition is applied at the centerline. The deposition of particles of three different sizes, which are 10, 50, and 75 microns, on ribbed and flat channel surfaces, are tested for $u_{\max }$ of 3,8 , and $13 \mathrm{~cm} / \mathrm{s}$. The Reynolds numbers of the channel flow, the particle Reynolds numbers, and the particle relaxation time are listed in Table 1 . The corresponding critical approaching velocities are calculated to be $0.36,0.079$, and $0.048 \mathrm{~cm} / \mathrm{s}$ for particles with diameters of 10,50 , and 75 microns, respectively. A particle is considered adhering to the surface if its velocity normal to the surface is lower than the critical velocity upon contact. The fluid and particle properties, geometrical parameters, and simulation parameters are given in Table 1.

Complex surface textures can induce instability even at low velocities. To capture the smallest structure of the hydrodynamics near the surface, very fine meshes that meet the requirement of Direct Numerical Simulation (DNS) are used in this study. In DNS, the mesh size $\Delta x$ and time step $\Delta t$ can be estimated as [42]:

$$
\Delta x \sim(R e)^{-3 / 4} L \text { and } \Delta t \sim \Delta x / U
$$

where $L$ is the characteristic length of the problem and $U$ is the velocity. Based on the velocity of $13 \mathrm{~cm} / \mathrm{s}$ and the channel height of $0.02 \mathrm{~m}, \Delta x$ is approximately 50 microns and $\Delta t$ is $300 \mu \mathrm{s}$. In this study, a maximum mesh size of 40 microns is used in a $1 \mathrm{~mm}$ thick layer above the surface as shown in Figure 2. Outside of this near surface layer, the maximum mesh size is 100 microns. The total number of elements in the near surface layer is 150,000 . Further reduction of mesh size by $50 \%$ yields $0.1 \%$ difference in the velocity at the location of $0.1 \mathrm{~mm}$ above the apex of a rib. A time step of $\Delta t<300 \mu \mathrm{s}$ is used in the transient simulation of the fluid flow before a steady state velocity field is established.

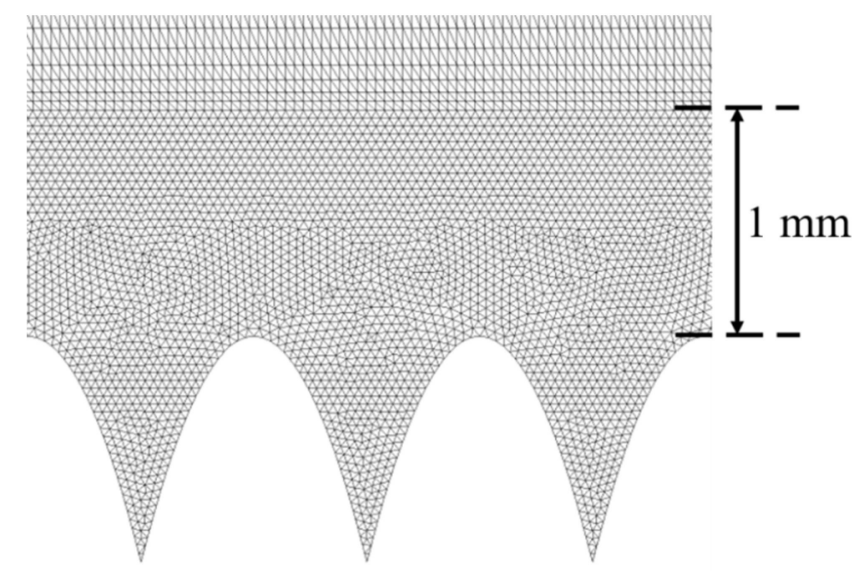

Figure 2. Mesh near the rib surface.

Well-mixed particle-laden fluids are used in many studies of particle transport and deposition in channel flows. To ensure a nearly uniform particle concentration, a flowrate-weighted particle distribution is applied when injecting particles from the inlet. However, this approach is not employed in this study because of the dependence of particle placement over the inlet on the flow velocity and the velocity profile. As our focus in the current study is how surface features affect the attachment of the particles, we aim to conduct numerical tests that highlight the effect of surface structures while minimizing the influence of other factors such as initial placement of the particles and particle concentrations. In light of this consideration, 1000 particles that are uniformly distributed in space are 
injected from the inlet of the channel every $0.5 \mathrm{~s}$ for $150 \mathrm{~s}$ in the numerical tests. Further increasing the number of the injected particles to 2000 every $0.5 \mathrm{~s}$ leads to less than $1 \%$ difference in the fraction of the particles that adhere to the surface. A time step of $10^{-4} \mathrm{~s}$ is chosen for the numerical integration of Equation (3). Further halving the time step yields a less than $0.1 \%$ difference in the fraction of particle deposition. A second-order implicit scheme is used to integrate Equation (3) for particle velocity and location.

Considering the assumption of a low particle concentration, a one-way coupling scheme is used in this study. The steady-state velocity field is obtained first, followed by the calculation of the acceleration, the velocity, and the location of each particle at each time step using Equation (3). For the numerical study of fluid flow, the pressure-velocity coupling scheme is used for pressure and momentum equation and the second-order upwind scheme is used for discretization. The simulation is performed using the software ANSYS-Fluent with user-defined subroutines or functions to implement additional forces for particle-surface interactions.

\section{Results}

In this study, simulations of fluid flow and particle attachment have been performed in a two-dimensional (2D) channel illustrated in Figure 1a with surface topographies shown in Figure 1b. The ribs shown in Figure $1 \mathrm{~b}$ share the same height $(H)$ with various pitches $(\lambda)$. To provide a reference for the ribbed surfaces, simulations are also conducted in the 2D channel with a flat test surface. This reference channel has a height of $h-2 H$ as the area for the fluid flow in the ribbed channel is reduced. Figure 3 shows the complex flow patterns near the arc-shaped ribs of different $\lambda / H$ ratios. The velocity fields demonstrate vortices formed between adjacent ribs and a wavy pattern of shear flows above the surface. Both the height of the vortex and the amplitude of the wavy flow are dependent on the $\lambda / H$ ratio of the ribs.

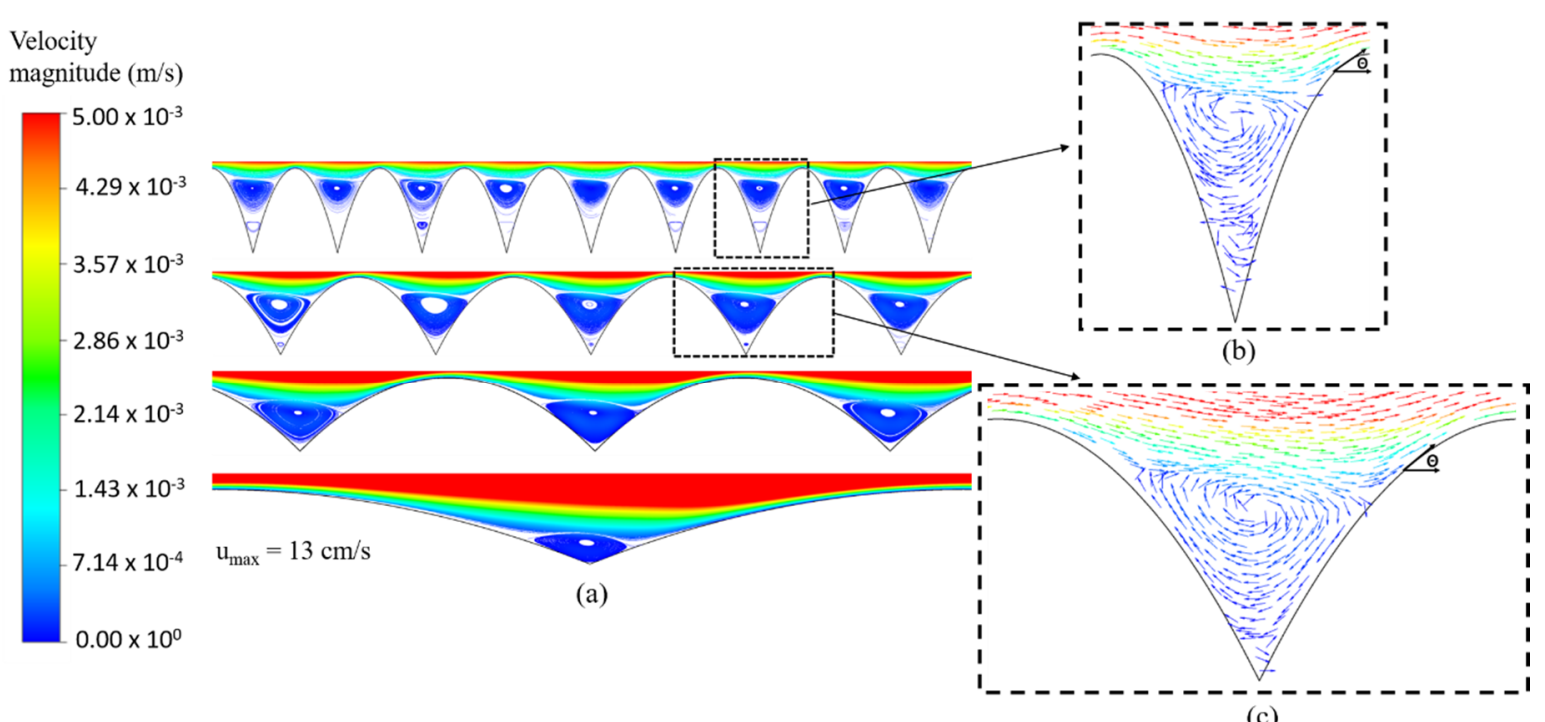

(c)

Figure 3. (a) Wavy flows near the surface at $13 \mathrm{~cm} / \mathrm{s}$; (b) and (c) velocity fields between adjacent ribs for pattern $1(\lambda / H=1)$ and pattern $2(\lambda / H=2)$, respectively. $\Theta$ is the angle of attack on the front side of a rib.

The shear rate distributions over the ribbed surfaces at a velocity of $8 \mathrm{~cm} / \mathrm{s}$ are shown in Figure 4a. The maximum shear rates generated by these surfaces at velocities ranging from 3 to $13 \mathrm{~cm} / \mathrm{s}$ are compared in Figure $4 \mathrm{~b}$. While the highest shear rate all occurs at the apex of the arc-shaped ribs, its magnitude varies over a large range despite the same height of the ribs. The ribs with a $\lambda / H=2$ is identified to yield the highest maximum shear rate. Additionally, the shear rate enhancement is more pronounced at high velocities as evidenced by the slopes of the plots in Figure $4 \mathrm{~b}$. 


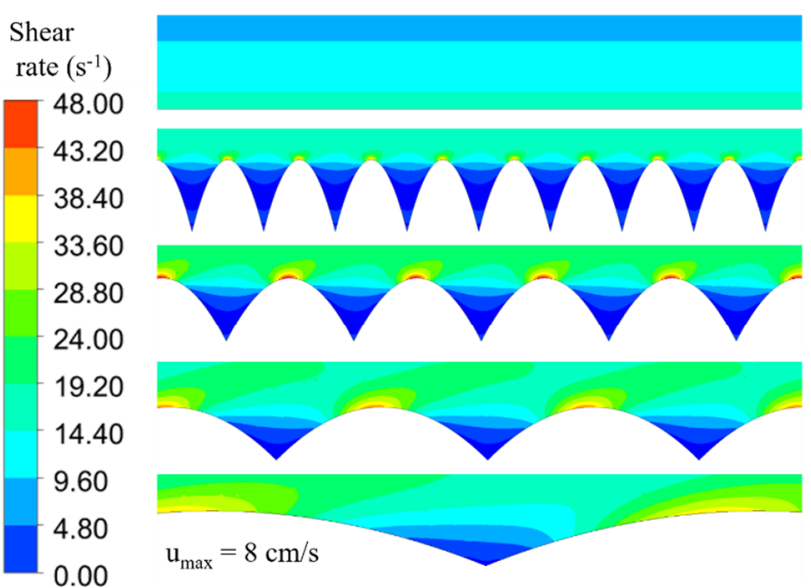

(a)

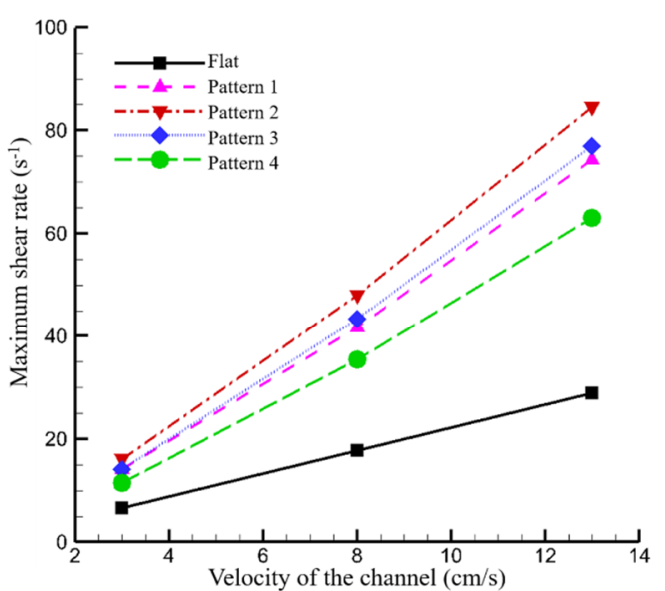

(b)

Figure 4. (a) Shear rate distributions over the ribbed and flat surfaces at $8 \mathrm{~cm} / \mathrm{s}$ and (b) the maximum shear rate versus velocity.

In this study, particle deposition is measured by the fraction of particle attachment, $f$, defined as the fraction of the injected particles that adheres to the test surface. At a low velocity of $3 \mathrm{~cm} / \mathrm{s}$, the ribs show negligible effect on the particle attachment regardless of particle size, as observed in Figure $5 \mathrm{a}$. At a higher velocity of $8 \mathrm{~cm} / \mathrm{s}$, the $f$ values for 50 and 75 micron particles decline on the ribs as displayed in Figure $5 \mathrm{~b}$. The attachment of 50 and 75 micron particles is further reduced by the ribs when the velocity is increased to $13 \mathrm{~cm} / \mathrm{s}$ as shown in Figure 5c. Interestingly, 10 micron particles remain insensitive to the ribbed surface topographies even at the higher velocities. The reduction in particle attachment is dependent on both the velocity and the particle size.
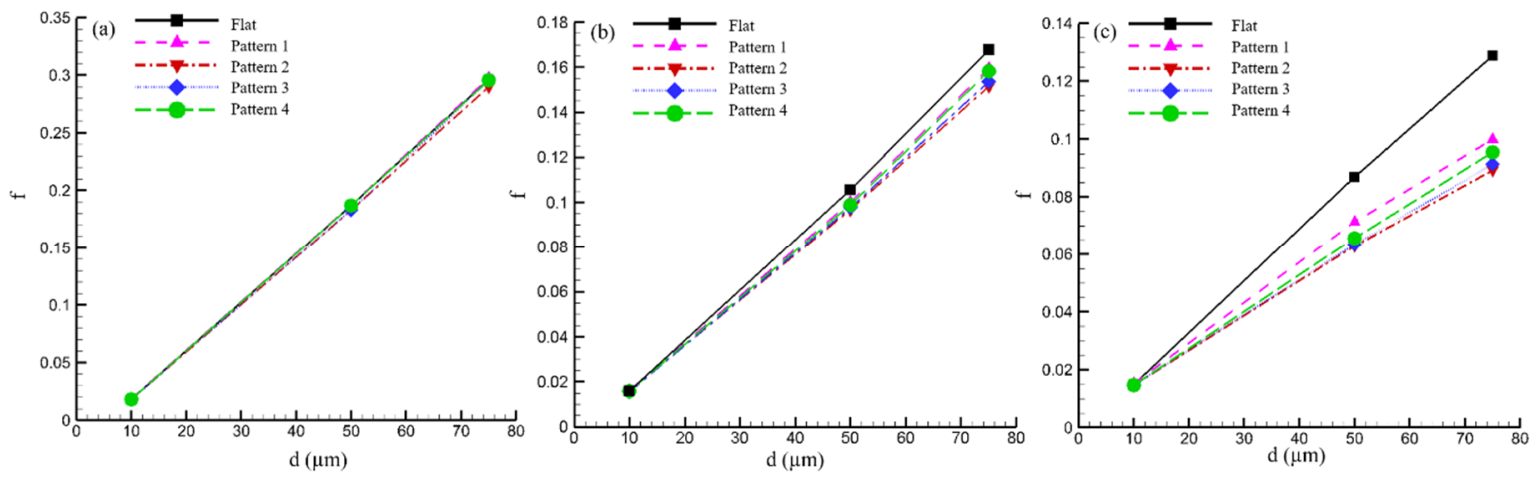

Figure 5. The fraction of particle attachment, $f$ for different ribbed surfaces at various fluid velocities of (a) $3 \mathrm{~cm} / \mathrm{s}$, (b) $8 \mathrm{~cm} / \mathrm{s}$, and (c) $13 \mathrm{~cm} / \mathrm{s}$.

Among the four ribbed surfaces, the rib geometry with a $\lambda / H=2$ is the most effective in reducing particle attachment for 50 and 75 micron particles as shown in Figure 6. Compared with the flat surface, this rib geometry reduces particle attachment for 50 and 75 micron particles, respectively, by $8.62 \%$ and $9.54 \%$ at the velocity of $8 \mathrm{~cm} / \mathrm{s}$, and by $27.6 \%$ and $30.54 \%$ at a velocity of $13 \mathrm{~cm} / \mathrm{s}$. Additionally, the simulation results show that regardless of surface topographies, particle attachment decreases with increasing velocity and decreasing particle size. This observation agrees with other theoretical and experimental studies [43]. 


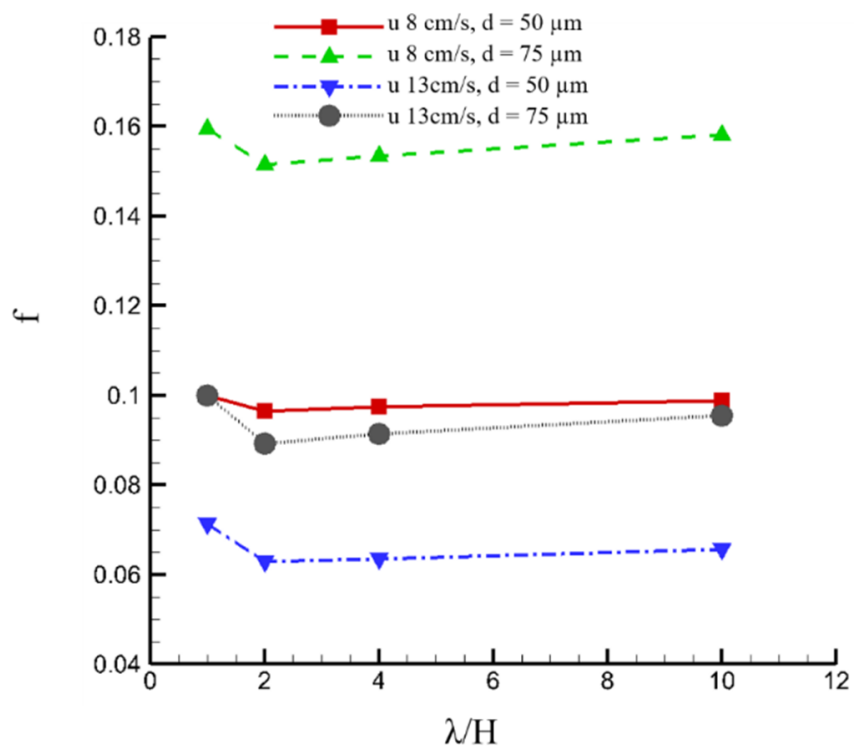

Figure 6. The fraction of particle attachment, $f$ for the ribbed surfaces of various $\lambda / H$ ratios.

We have also performed simulation of particle attachment to ribbed surfaces with the rib height $(H)$ of $0.5 \mathrm{~mm}$ and $\lambda / H$ ratios of $1,2,4$, and 10 . Again, the surface with a $\lambda / H=2$ demonstrates the highest shear rate enhancement shown in Figure $7 \mathrm{a}$ and the lowest fraction of particle attachment. However, the reduction in particle attachment is not as effective as the $1 \mathrm{~mm}$-height ribs of the same $\lambda / H$ ratio, as displayed in Figure $7 \mathrm{~b}$.
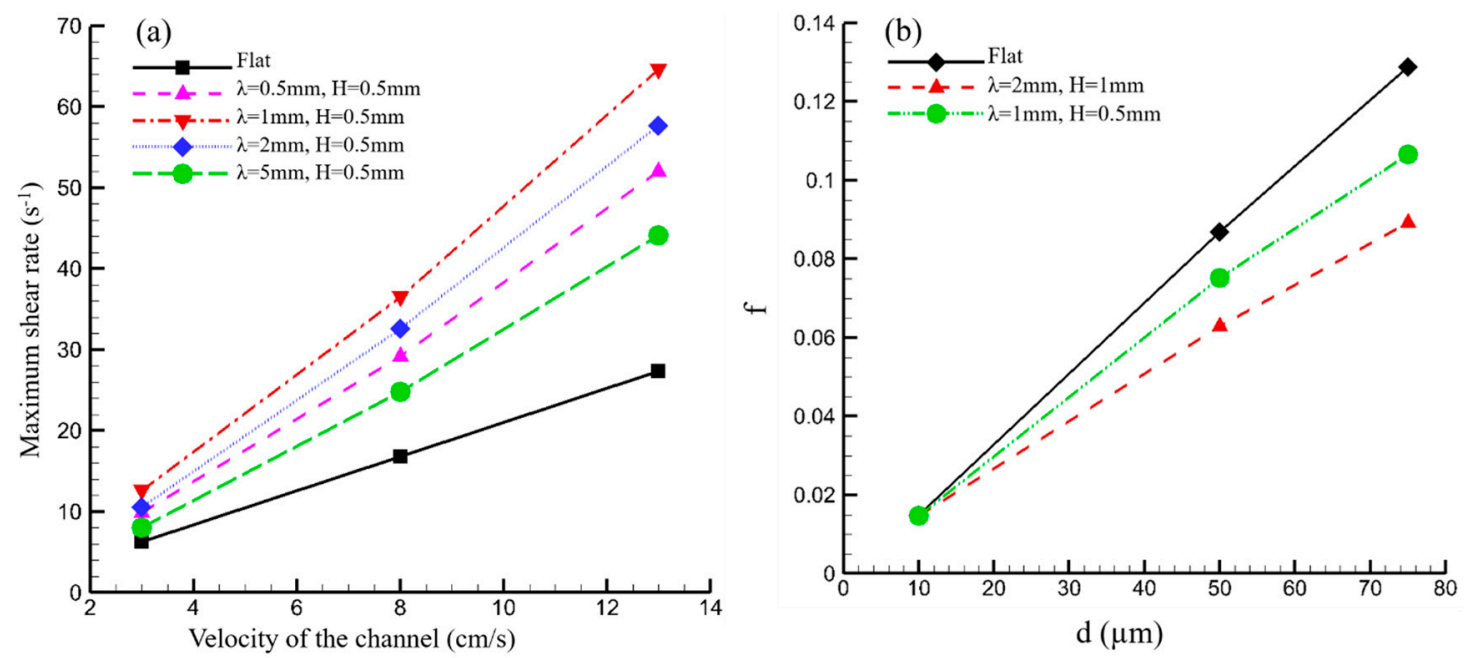

Figure 7. (a) Maximum shear rate versus velocity for different ribbed surfaces with $H=0.5 \mathrm{~mm}$, and (b) the fractions of particle attachment at $13 \mathrm{~cm} / \mathrm{s}$ for the flat surface and the ribbed surfaces with a $\lambda / H=2$ and the rib heights of $1 \mathrm{~mm}$ and $0.5 \mathrm{~mm}$.

The distributions of 50 and 75 micron particles are also characterized on the arc-shaped ribs with a $\lambda / H$ value of 2 . As shown in Figure 8 , a unit surface structure can be divided by a midline into two sub-areas: apex and valley. The total number of particles that attach to each of the sub-areas over the entire test surface is calculated and compared for different velocities. Figure 8 shows that at $8 \mathrm{~cm} / \mathrm{s}$, the amount of 50 micron particles settled in the valley is slightly higher than that in the apex. In contrast, most 75 micron particles fall in the valley at $13 \mathrm{~cm} / \mathrm{s}$. The distribution of the larger particles in the high velocity flow is more sensitive to the surface topography. 


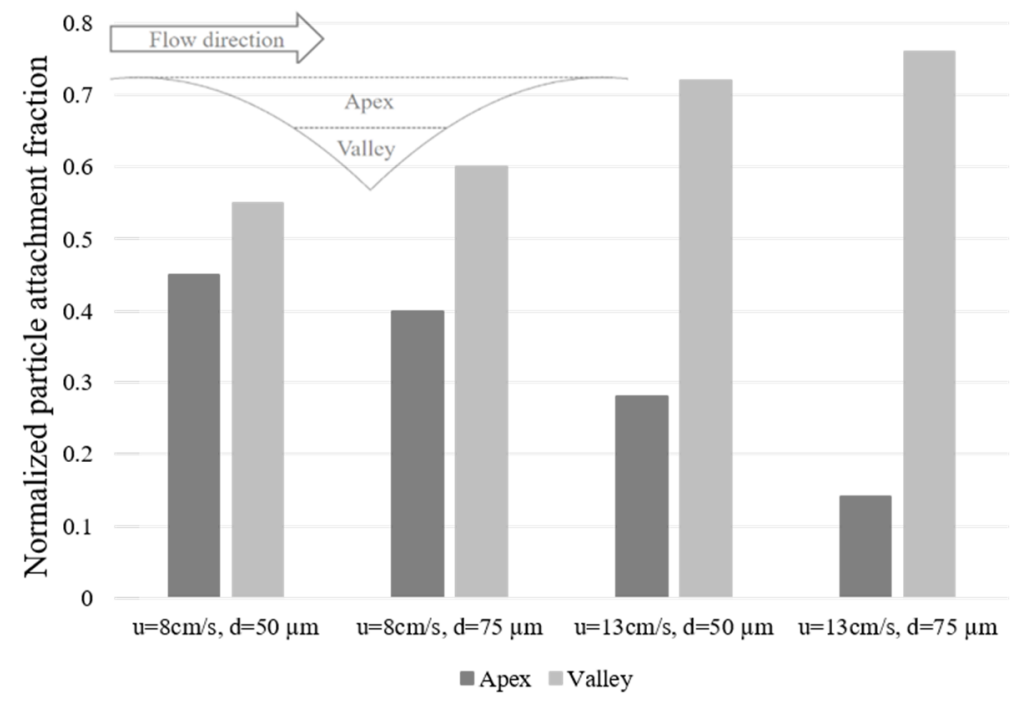

Figure 8. Normalized fractions of particles adhered to apex and valley areas for pattern $2(\lambda=2 \mathrm{~mm}$ and $H=1 \mathrm{~mm}$ ).

\section{Discussion}

The behavior of particle attachment to a surface is determined by the complicated interplay between the particle, the hydrodynamics, and the surface topography. When a stream of liquid is passed over a ribbed surface, complex hydrodynamics is created even at moderate flow velocities. The enhanced shear rate, the flow pattern, the contact geometry, and the particle size are important factors that determine the fraction of particle attachment to the surface as well as the particle distribution over the surface.

Our simulation shows that the maximum shear rates generated at the ribbed surfaces are significantly higher than the flat surface under an identical flow velocity. As the height of the flat-surfaced channel is reduced, the observed shear enhancement can only be attributed to the wavy flow and the surface topography illustrated in Figure 3. As the fluid follows the contour of the ribs, the upward acceleration of the fluid along the front side of the rib maximizes the shear rate near the apex. The magnitude of the shear is dependent on both the wavy flow amplitude and the angle of attack at the front of the rib. For the surface with a $\lambda / H=1$, the close spacing between the ribs results in a small wavy flow amplitude and a limited amount of the fluid interacting with the ribs. In the opposite case of a large $\lambda / H=10$, the effect of the large wave amplitude is weakened by the small slope of the rib surface. With these competing mechanisms at play, the surface topography with a $\lambda / H=2$ yields the highest maximum shear rate for all velocities. When the height of the arc-shaped ribs is reduced to $0.5 \mathrm{~mm}$, the highest shear rate is again yielded at a $\lambda / H$ value of 2 , illustrating the same mechanisms at play. To further improve the shear rate, topographical parameters that can suppress the stagnant zones between the ribs while maintaining an optimal angle of attack should be developed in the future study.

High shear rates near a surface will reduce the fraction of particle attachment. However, this is only observed in our study for 50 and 75 micron particles at the velocities of 8 and $13 \mathrm{~cm} / \mathrm{s}$. This discrepancy can be explained by the threshold shear rate. For deposition of micrometer-sized particles in laminar flows, the van der Waals force only dominates over a short particle-surface distance of hundreds of nanometers. Beyond this distance, it has negligible influence on the particle trajectory. The role of the Saffman force in laminar flows is also insignificant. Thereby, the motion of the particles is dictated by the competition between the magnitudes of the buoyancy force, $\mathbf{F}_{g}$, and the wall-induced lift forces, $\mathbf{F}_{L}$, 
in the direction normal to the surface. A particle will move away from the surface when the lift force overpowers gravity. The ratio of the magnitudes of these two forces near a surface is expressed as:

$$
\left|\frac{\mathrm{F}_{L}}{\mathrm{~F}_{g}}\right|=\frac{6 f_{L} \gamma^{2} d_{p} \rho_{f}}{\pi\left(1-\frac{\rho_{f}}{\rho_{p}}\right) g \pi \rho_{p}}
$$

Based on $\left|F_{L} / F_{g}\right|<1$, a threshold shear rate, $\gamma_{c r}$, can be calculated. The threshold shear rate for polystyrene particles of various diameters in water is given in Figure 9. For 10 micron particles, the threshold shear rate is $140 \mathrm{~s}^{-1}$, higher than any maximum shear rate presented in Figure 4 . This explains the reason why 10 micron particles are insensitive to increasing velocities and surface topographies. On the other hand, for the lowest fluid velocity of $3 \mathrm{~cm} / \mathrm{s}$, the ribbed surfaces are unable to raise shear rates above the respective threshold values for all three particles sizes; therefore, no reduction in the particle attachment is observed.

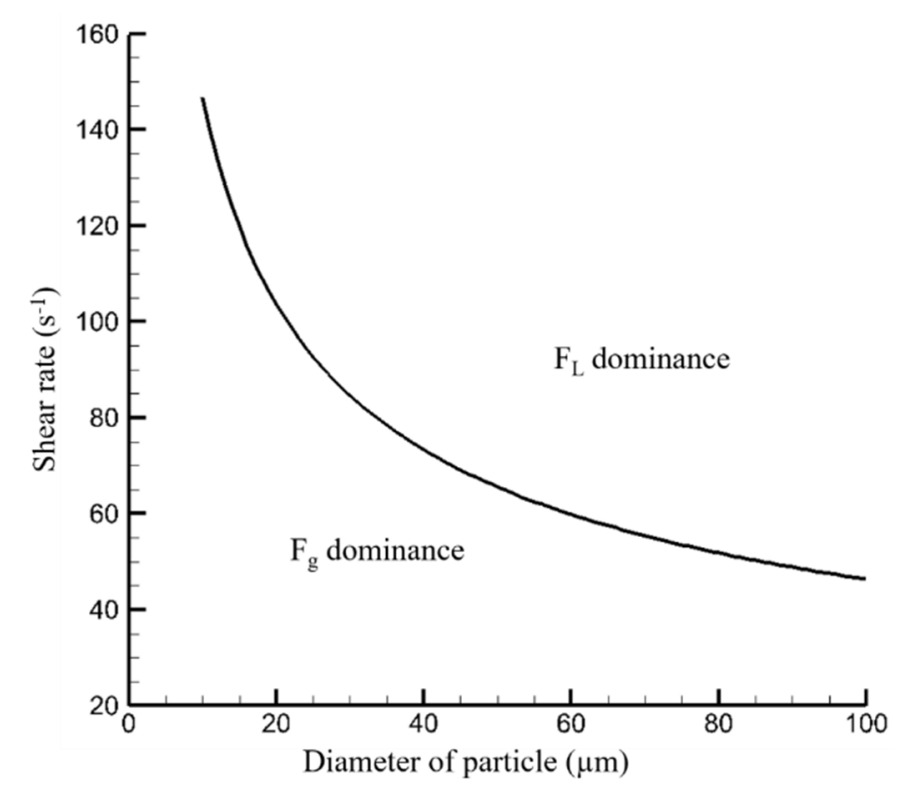

Figure 9. Threshold shear rate for polystyrene particles in an aqueous suspension.

The reduction in the particle attachment by the ribs is most effective for large particles at high velocities due to the decline in threshold shear rates and the nonlinear dependence of the magnitude of $\mathbf{F}_{L}$ on the shear rate $\left(F_{L} \propto \gamma^{2}\right)$. Considering the pivotal role of the shear rate in particle adherence to a surface, it is not surprising that the ribbed surface yielding the highest maximum shear rate is associated with the most reduction in particle attachment.

Besides the reduced particle attachment to the surface, a non-uniform shear rate also affects the distribution of the attached particles on the surface. While it has been established that more particles fall in the valley area than the apex, our study shows that the disproportionality is very sensitive to particle size and velocity. With more particles accumulating in the valley, the adhesive particle-surface interaction will be replaced by the cohesive particle-particle interactions. This change has significant influence on the evolution of particle deposition rate with time. Quantitative information on the particle distribution over a rib is also very helpful for understanding particle resuspension from the surface.

One limitation of our numerical models is the idealized representation of real situations such as spherical and rigid particles, two-dimensional laminar flows, zero electrostatic forces, and no energy loss with collision. While these assumptions may compromise the accuracy of the predicted particle fouling rate, the results can still elucidate the physics underlying the particle-rib interactions and the effect of surface topographical features on particle attachment. Such understanding and knowledge is 
still valuable for surface texture choice and design. In addition, this study focuses only on particle attachment to clean surfaces, and the effect of particle interaction with the previously attached particle and the change of surface topographies by particle accumulation is not considered. Research effort will be continued to study particle deposition with particle-particle interactions, altered contact geometry by particle attachment, and the electrostatic force.

\section{Conclusions}

Numerical simulations have been performed to study the effect of surface topography on particle attachment in a two-dimensional channel flow. The ribs on the surface can significantly enhance the shear rates. The shear rate enhancement can be attributed to the wavy flows over the ribbed surface and the weak vortices between adjacent ribs. For arc-shaped ribs of identical height, varying pitch-to-height ratios, $\lambda / H$, can alter the amplitude of the wavy flow and the angle of attack of the fluid on the ribs. As a result, the rib geometry with $\lambda / H=2$ demonstrates the greatest shear rate near the rib apex. As the wall-induced lift force is nonlinearly dependent on $\gamma$, this surface geometry yields the lowest fraction of particle attachment. However, the ribbed surfaces show negligible effects in two cases. One is the particle attachment from channel flow at low velocity, such as $3 \mathrm{~cm} / \mathrm{s}$, and the other is small particle diameter such as 10 micron particles. Analysis of forces acting on the particles identifies a threshold shear rate to reduce the particle attachment. Non-flat surface geometries lead to non-uniform distribution of particles, and the non-uniformity is more pronounced for larger particles and higher flow velocities. Understanding of the effect of surface topography on particle attachment will benefit design of surface textures for mitigating particulate fouling.

Author Contributions: Conceptualization and planning of the work presented in the manuscript was conducted by all contributing authors. R.M. contributed to methodology and M.M.Z. contributed to all CFD simulations. Data analysis was done by R.M. and M.M.Z. R.M. and L.Z. supervised and edited the final draft of the manuscript. All authors have read and agreed to the published version of the manuscript.

Funding: This research was supported by an NSF grant (CBET-1705538).

Conflicts of Interest: The authors declare no conflict of interest.

\section{References}

1. Epstein, N. Particulate Fouling of Heat Transfer Surfaces: Mechanisms and Models. NATO ASI Ser. 1988, 145, 143-164. [CrossRef]

2. Epstein, N. Elements of particle deposition onto nonporous solid surface parallel to suspension flows. Exp. Therm. Fluid Sci. 1997, 14, 323-334. [CrossRef]

3. Yiantsios, S.G.; Karabelas, A. Deposition of micron-sized particles on flat surfaces: Effects of hydrodynamic and physicochemical conditions on particle attachment efficiency. Chem. Eng. Sci. 2003, 58, 3105-3113. [CrossRef]

4. Guha, A. Transport and deposition of particles in turbulent and laminar flow. Annu. Rev. Fluid Mech. 2008, 40, 311-341. [CrossRef]

5. Henry, C.; Minier, J. Progress in particle resuspension from rough surfaces by turbulent flows. Prgo. Energy Combust. 2014, 45, 1-53. [CrossRef]

6. Kim, N.-H.; Webb, R.L. Particulate fouling of water in tubes having a two-dimensional roughness geometry. Int. J. Heat Mass Transf. 1991, 34, 2727-2738. [CrossRef]

7. Webb, R.L.; Kim, N.-H. Principles of Enhanced Heat Transfer, 2nd ed.; Taylor \& Francis: New York, NY, USA, 2005; ISBN 978-1591690146.

8. Somerscales, E.F.C.; Ponteduro, A.F.; Bergles, A.E. Particulate fouling of heat transfer tubes enhanced on their inner surface. ASME HTD 1991, 164, 17-28.

9. Somerscales, E.F.C.; Bergles, A.E. Enhancement of Heat Transfer and Fouling Mitigation. Adv. Heat Transf. 1997, 30, 197-253. [CrossRef] 
10. Pääkkönen, T.M.; Ojaniemi, U.; Riihimäki, M.; Muurinen, E.; Simonson, C.J.; Keiski, R. Surface patterning of stainless steel in prevention of fouling in heat transfer equipment. Mater. Sci. Forum 2013, 762, 493-500. [CrossRef]

11. Pääkkönen, T.M.; Ojaniemi, U.; Pättikangas, T.; Manninen, M.; Muurinen, E.; Keiski, R.; Simonson, C.J. CFD modelling of $\mathrm{CaCO} 3$ crystallization fouling on heat transfer surfaces. Int. J. Heat Mass Transf. 2016, 97, 618-630. [CrossRef]

12. Watkinson, A.P. Interactions of enhancement and fouling. In Fouling and Enhancement Interactions; Rabas, T.J., Chenoweth, J.M., Eds.; ASME: New York, NY, USA, 1991; Volume 164, pp. 1-7.

13. Li, W. Modeling liquid-side particulate fouling in internal helical-rib tubes. Chem. Eng. Sci. 2007, 62, 4204-4213. [CrossRef]

14. Magin, C.M.; Cooper, S.P.; Brennan, A.B. Non-toxic anti-fouling strategies. Mater. Today 2010, 13, 36-44. [CrossRef]

15. Lu, H.; Lu, L. Effects of rib spacing and height on particle deposition in ribbed duct airflows. Build Environ. 2015, 92, 317-327. [CrossRef]

16. Lu, H.; Lu, L. A numerical study of particle deposition in ribbed duct flow with different rib shapes. Build Environ. 2015, 94, 43-53. [CrossRef]

17. Lu, H.; Lu, L. CFD investigation on particle deposition in aligned and staggered ribbed duct air flows. Appl. Therm. Eng. 2016, 93, 697-706. [CrossRef]

18. Lu, H.; Lu, L. Investigation of particle deposition efficiency enhancement in turbulent duct air flow by surface ribs with hybrid-size ribs. Indoor Built Environ. 2017, 26, 806-820. [CrossRef]

19. Hong, W.; Wang, X.; Zheng, J. Numerical study on particle deposition in rough channels with different structure parameters of rough elements. Adv. Powder Technol. 2018, 29, 2895-2903. [CrossRef]

20. Xu, H.; Fu, S.C.; Wing, T.L.; Lai, T.W.; Chao, C.Y.H. Enhancement of submicron particle deposition on a semi-circular surface in turbulent flow. Indoor Built Environ. 2019, 1-16. [CrossRef]

21. Kasper, R.; Turnow, J.; Kornev, N. Numerical Modeling and simulation of particulate fouling of structured heat transfer surfaces using a multiphase Euler-Lagrange approach. Int. J. Heat Mass Transf. 2017, 115, 932-945. [CrossRef]

22. Kasper, R.; Deponte, H.; Michel, A.; Turnow, J.; Augustin, W.; Scholl, S.; Kornev, N. Numerical investigation of the interaction between local flow structures and particulate fouling on structured heat transfer surfaces. Int. J. Heat Fluid Flow 2018, 71, 68-79. [CrossRef]

23. Sommerfeld, M.; Wirth, K.-E.; Muschelknautz, U. L3 two-phase gas-solid flow. VDI Heat Atlas 2010, 1181-1238. [CrossRef]

24. Putnam, A. Integrable form of droplet drag coefficient. ARS J. 1961, 31, 1467-1470.

25. Saffman, P.G. The lift on a small sphere in a slow shear flow. J. Fluid Mech. 1965, 22, 385-400. [CrossRef]

26. Saffman, P.G. The lift on a small sphere in a slow shear flow-corrigendum. J. Fluid Mech. 1968, 31, $624-625$. [CrossRef]

27. Mei, R. An approximate expression for the shear lift force on a spherical particle at finite Reynolds number. Int. J. Multiph. Flow 1992, 18, 145-147. [CrossRef]

28. Asmolov, E.S. The inertial lift on a spherical particle in a plane Poiseuille flow at large channel Reynolds number. J. Fluid Mech. 1999, 381, 63-87. [CrossRef]

29. Carlo, D.D.; Irimia, D.; Tompkins, R.G.; Toner, M. Continuous inertial focusing, ordering, and separation of particles in microchannels. Proc. Natl. Acad. Sci. USA 2007, 104, 18892-18897. [CrossRef]

30. Derjaguin, B.; Landau, L. Theory of the stability of strongly charged lyophobic sols and of the adhesion of strongly charged particles in solutions of electrolytes. Prog. Surf. Sci. 1993, 43, 30-59. [CrossRef]

31. Verwey, E.; Overbeek, J. Theory of the Stability of Lyophobic Colloids. Dover Publ. Inc. 1999, 51, $631-636$. [CrossRef]

32. Russel, W.; Saville, A.D.; Schowalter, W. Colloidal Dispersions; Cambridge University Press: Cambridge, UK, 1989; ISBN 0521341884.

33. Lefevre, G.; Jolivet, A. Calculation of Hamakar Constant applied to the Deposition of Metallic oxide Particles at High Temperature. In Proceedings of the International Conference on Heat Exchanger Fouling and Cleaning VIII-2009, Schladming, Austria, 14-19 June 2009.

34. Israelachvili, J. Intermolecular and Surface Forces, 3rd ed.; Academic Press, University of California Santa Barbara: California, CA, USA, 2011; ISBN 978-0-12-375182-9. 
35. Leitc, F.L.; Bueno, C.C.; Da Roz, A.L.; Ziemath, E.C.; Oliveira, O.N. Theoretical models for surface forces and adhesion and their measurement using atomic force microscopy. Int. J. Mol. Sci. 2012, 13, 12773-12856. [CrossRef]

36. Abd-Elhady, M.S.; MRindt, C.C.; Wijers, J.G.; Steenhoven, A.A.; Bramer, E.A.; Meer, T.H. Minimum gas speed in heat exchangers to avoid particulate fouling. Int. J. Heat Mass Transf. 2004, 47, 3943-3955. [CrossRef]

37. Heinl, E.; Bohnet, M. Calculation of particle wall adhesion in horizontal gas-solids flow using CFD. Powder Technol. 2005, 159, 95-104. [CrossRef]

38. Löffler, F.; Muhr, W. Die Abscheidung von Feststoffteilchen und Tropfen an Kreiszylinderninfolge von Trägheitskräften. Chem. Ing. Tech. 1972, 44, 510-514. [CrossRef]

39. Cengel, Y.A.; Cimbala, J.M. Fluid Mechanics Fundamentals and Applications, 2nd ed.; McGraw Hill: New York, NY, USA, 2010; ISBN 978-0-07-352926-4.

40. Mallick, P.K.; Zhou, Y. Yield and fatigue behavior of polypropylene and polyamide-6 nanocomposites. J. Mater. Sci. 2003, 38, 3183-3190. [CrossRef]

41. Van Oss, C.J.; Chaudhury, M.K.; Good, R.J. Interfacial Lifshitz-van der Waals and polar interactions in macroscopic systems. Chem. Rev. 1998, 88, 927-941. [CrossRef]

42. Moin, P.; Mahesh, K. Direct Numerical Simulation: A Tool in Turbulence Research. Annu. Rev. Fluid Mech. 1998, 30, 539-578. [CrossRef]

43. Chamra, L.M.; Webb, R.L. Modeling liquid-side particulate fouling in enhanced tubes. Int. J. Heat Mass Transf. 1994, 37, 571-579. [CrossRef]

(C) 2020 by the authors. Licensee MDPI, Basel, Switzerland. This article is an open access article distributed under the terms and conditions of the Creative Commons Attribution (CC BY) license (http://creativecommons.org/licenses/by/4.0/). 